\title{
Editorial
}

\section{Válvula aórtica bicúspide y dilatación de la raíz de la aorta o de la aorta ascendente}

\author{
Ernesto Larraín R.
}

Cirugía Cardíaca

Clínica Santa María y Clínica Dávila

\section{Resumen:}

La dilatación de la aorta ascendente es un acompañante muy frecuente de la valvulopatía aórtica bicúspide, y a su vez la cardiopatía congénita más frecuente. Entre las teorías acerca de su origen, la más establecida es la que la liga al efecto de metaloproteinasas sobre la pared arterial. En este editorial se hace referencia a la publicación simultánea en este número del artículo de Dayan et al., que describen la evolución de la dilatación aórtica post reemplazo valvular. 


\section{Dilatation of the aortic root and the ascending aorta in patients with bicuspid aortic valve disease}

Aortic root and ascending aorta dilatacion is a common consequence of bicsupid aortic valve disease, in turn the most frequent form of congenital heart disease. Among the different theories proposed to explain this finding, the action of metallo proteinases upon the aor- tic wall seems to be well established. In this Editorial, the concurrent work by Dayan et al published in this issue, dealing with aortic root dilatation post aortic valve replacement in patients with bicuspid aortic valve is analyzed.
La condición de válvula aórtica bicúspide es la malformación congénita más frecuente en la especia humana con una frecuencia estimada entre 1 y $2 \%$ de la población. Existe una relación bien conocida entre válvula aórtica bicúspide y dilatación de la raíz de la aorta y aorta ascendente, la que actualmente se atribuye a la acción de enzimas proteolíticas llamadas metaloproteinasas 2 y 9 , que degradan la matriz extracelular y debilitan las paredes de la aorta a ese nivel ${ }^{1,2,3,4}$. Esto ocurre en una cantidad significativa de los pacientes portadores de una válvula aórtica bicúspide (VAB), los que van a requerir de una intervención quirúrgica tal de corregir su patología valvular, su dilatación aórtica, o ambas, en alguna etapa de sus vidas.

En esta edición, Dayan y colaboradores ${ }^{5}$ sostienen que habría una cierta acción genética sobre la pared de la aorta como la explicación para su dilatación en pacientes con VAB; la teoría hemodinámica a mi parecer, ha sido descartada hace ya varios años como causal de esta condición patológica $^{6,7}$.

Por otra parte, la condición de VAB y su relación hereditaria a la fecha no es algo que se haya establecido con claridad $^{8,9}$ y existe información contradictoria en tal sentido, pero bien podría ser una causal.

Por otra parte y debido a los mecanismos patológicos involucrados en la dilatación de la raíz aórtica como de la porción tubular de la misma, se hace evidente que estas porciones de la aorta seguirán dilatándose en pacientes portadores de VAB una vez que la válvula aórtica ha sido reemplazada ${ }^{10,11}$.

Esto ha llevado a algunos autores a sugerir el reemplazo de la aorta en pacientes con VAB al momento del reemplazo valvular aórtico incluso en casos de dilatación modera$\mathrm{da}^{10,12}$. Es nuestro parecer que en pacientes con diámetros superiores a los $46 \mathrm{~mm}$ y existiendo las condiciones de un bajo riesgo quirúrgico otorgado por un grupo quirúrgico con experiencia en esta patología es razonable no esperar a que la aorta llegue a los $55 \mathrm{~mm}$ y así proceder al reemplazo de la aorta ascendente o de la raíz si así procede. Esto evitaría una reoperación en los años por venir.

La experiencia y el control de los pacientes con ecocardiografía indican que el efecto de la aortorrafia sobre el diámetro de la aorta en el seguimiento alejado de estos pacientes juega un rol marginal y un número significativo de estos pacientes va a dilatar su aorta ascendente/raíz en el tiempo de igual forma.

En cuanto al tratamiento médico, se ha podido comprobar en pacientes portadores de Síndrome de Marfán, que el tratamiento farmacológico con beta bloqueadores no reduce el ritmo de progresión de la dilatación en pacientes pero sí el tratamiento con bloqueadores de la angiotensina II $^{4,13}$. No hay estudios que extrapolen ésto a pacientes con VAB y en la práctica clínica se utilizan ambas drogas en 
forma habitual.

Los medicamentos tipo estatinas se han demostrado beneficiosos también en pacientes portadores de VAB al limitar la dilatación de la aorta debido a su efecto reductor de la acción de las metaloproteinasas y al mejorar la función endotelial al aumentar la sintetasa del óxido nítrico ${ }^{14}$. A la luz del conocimiento actual, es absolutamente esperable que una porción significativa de éstos pacientes presenten progresiva dilatación de la raíz aórtica, pero también dilatación de la aorta ascendente (en su porción tubular) durante el seguimiento de algunos años.

Es llamativo que los datos aportados en el estudio de Dayan y colaboradores, no revelan aumento en el tamaño de la porción tubular de la aorta en ninguno de los 23 casos de la serie ${ }^{5}$. En la práctica quirúrgica es habitual observar que un número significativo de pacientes portadores de una VAB van a presentar dilatación la raíz de la aorta, como también dilatación de la porción inicial de la aorta ascendente y en ocasiones la totalidad de la porción tubular de la aorta. Existe por otra parte la asociación, también de cierta frecuencia, en que los pacientes portadores de una VAB presentan dilatación sólo de la porción tubular o porción supracomisural de la aorta sin compromiso de la raíz de la aorta.

En cuanto al tamaño de la aorta, no es lo mismo en cuanto a su validez como medición aislada decir que un paciente de $1.5 \mathrm{mts} /{ }^{2}$ de superficie corporal tiene una aorta ascendente de $45 \mathrm{~mm}$ o que un paciente con una superficie cor- poral de $2 \mathrm{mts} /{ }^{2}$ tiene una aorta de $45 \mathrm{~mm}$; su pronóstico y opciones terapéuticas son muy distintas en cada caso. Es por esto que la validación de las medidas del diámetro transversal de la aorta deberían ser indexadas a la superficie corporal en metros cuadrados.

De aquí la pregunta: ¿qué es una aorta normal?, lo que algunos autores han intentado responder en base a estudios de muchos años de seguimiento de pacientes con dilatación de la aorta ascendente/raíz y su seguimiento alejado utilizando imágenes ${ }^{15,16}$.

En este seguimiento, debe tenerse particular cuidado con aquellos pacientes que aumentan sustancialmente su diámetro aórtico (mayor a $0.5 \mathrm{cms}$ ) entre controles y no sólo fijarse en el valor absoluto de la dilatación de la aorta.

De lo anterior, se desprende la importancia del seguimiento con imágenes, tanto ecocardiográfica como de escaner, en este grupo de pacientes que presentan alto riesgo de rotura y/o disección de la aorta ascendente. Lo ideal es realizar controles anuales.

Los antecedentes familiares de patología de la aorta (raíz o aorta ascendente) por su parte, siempre son uno de los factores más importantes a considerar y deben ser atentamente solicitados caso a caso. Esto define, en algunos casos con fuertes antecedentes familiares de rotura o de disección aórtica aguda, la oportunidad de su reemplazo incluso con valores considerados como dilatación moderada (45 mm en diámetro).

\section{Bibliografía}

1. NATAATMADJA M, WEST M, WEST J, SUMMERS K, WALKER PH, NAGATA M, et al. Abnormal Extracellular Matrix Protein Transport Associated With Increased Apoptosis of Vascular Smooth Muscle Cells in Marfan Syndrome and Bicuspid Aortic Valve Thoracic Aortic Aneurysm. Circulation. 2003;108: II-329-II-334
2. FEDAK P, VERMA S, DAVID T, LEASK R, WEISEL R, BUTANY J. Clinical and Pathophysiological Implications of a Bicuspid Aortic Valve Circulation. 2002;106:900-904

3. LESAUSKAITE V, TANGANELLI P, SASSI C, NERI E, DICIOLLA F, IVANOVIENE L, et al. Smooth Muscle Cells of the Media in the Dilatative Pathology of Ascending Thoracic Aorta: Morphology, Immunoreactivity for Osteopontin, Matrix Metalloproteinases, and Their Inhibi- 
tors. Hum Pathol 2001;32:1003-1011

4. TADROS T, KLEIN M, SHAPIRA O. Ascending Aortic Dilatation Associated With Bicuspid Aortic Valve : Pathophysiology . Molecular Biology, and Clinical Implications. Circulation. 2009;119:880-890

5. Dilatación de la raíz aórtica en pacientes con válvula bicúspide tras la sustitución valvular aórtica Víctor Dayan, Leandro Cura, Nelson Pizzano, León Muñoz, Virginia Michelis, Enrique Domínguez, Gerardo Soca, Alejandro Ferreiro / hay que poner el numero de pagian del trabajo de Dayan

6. DE SA M, MOSHKOVITZ Y, BUTANY J, DAVID T. Histologic abnormalities of the ascending aorta and pulmonary trunk in patients with bicuspid aortic valve disease: clinical relevance to the ross procedure. J Thorac Cardiovasc Surg 1999;118:588-96.

7. BONDERMAN D, GHAREHBAGHI-SCHNELL E, WOLLENEK G, MAURER G, BAUMGARTNER H, LANG IM. Mechanisms Underlying Aortic Dilatation in Congenital Aortic Valve Malformation. Circulation. 1999;99:2138-2143.

8. VALLELY MP, SEMSARIAN C, BANNON PG. Management of the Ascending Aorta in Patients with Bicuspid Aortic Valve Disease. Heart Lung and Circulation 2008;17:357-363

9. FEDAK PW, VERMA S, DAVID TE, LEASK RL, WEISEL RD, BUTANY J. Clinical and Pathophysiological Implications of a Bicuspid Aortic Valve. Circulation. 2002;106:900-904
10. RUSSO C, MAZZETTI S, GARATTI A, RIBERA E, MILAZZO A, BRUSCHI G, et al. Aortic Complications After Bicuspid Aortic Valve Replacement: Long-Term Results Ann Thorac Surg 2002;74:S1773-6

11. YASUDA H, NAKATANI S, STUGAARD M, TSUJITAKURODA Y, BANDO K, KOBAYASHI J, et al. Failure to Prevent Progressive Dilation of Ascending Aorta by Aortic Valve Replacement in Patients With Bicuspid Aortic Valve: Comparison With Tricuspid Aortic Valve. Circulation. 2003;108:II-291-II-294

12. URBANSKI PP, WAGNER M, ZACHER M, HACKER RW. Aortic Root Replacement Versus Aortic Valve Replacement: A Case-Match Study Ann Thorac Surg 2001;72:28 -32

13. BROOKE BS, HABASHI JP, JUDGE DP, PATEL N, LOEYS B, DIETZ HC 3RD. Angiotensin II blockade and aortic-root dilation in Marfan's syndrome. N Engl J Med. 2008;358:2787-2795

14. VERMA S, SZMITKO PE, FEDAK PW, ERRETT L, LATTER DA, DAVID TE. Can statin therapy alter the natural history of bicuspid aortic valves? Am J Physiol. 2005;288:H2547-H2549

15. ROMAN MJ, DEVEREUX RB, KRAMER-FOX R, O'LOUGHLIN J. Two-dimensional echocardiographic aortic root dimensions in normal children and adults. American Journal of Cardiology 1989; 64:507-512

16. DAVIES RR, GALLO A, COADY MA, TELLIDES G, BOTTA DM, BURKE B, et al. Novel measurement of relative aortic size predicts rupture of thoracic aortic aneurysms. Ann Thorac Surg 2006; 81: 169 - 177 
BONE P, FETTERLY L, et al. Effect of behavioral management on quality of life in mild heart failure: a randomized controlled trial. Patient Educ Couns 2005;58:27-34.

24. MARTENSSON J, STRO "MBERG A, DAHLSTRO "MU, KARLSSON JE, FRIDLUND B. Patients with heart failure in primary health care: effects of a nurse-led intervention onhealth-related quality of life and depression. Eur J Heart Fail 2005;7:393-403.

25. WIERZCHOWIECKI M, POPRAWSKI K, NOWICKA A, KANDZIORA M, PIATKOWSKA A, JANKOWIAK $\mathrm{M}$, et al. A new programme of multidisciplinary care for patients with heart failure in Poznan: one-year follow-up. Kardiol Pol 2006;64:1063-70.

26. BOCCHI EA, CRUZ F, GUIMARAES G, PINHO MOREIRA LF, ISSA VS, AYUB FERREIRA SM, et al. Longterm prospective, randomized, controlled study using repetitive education at six-month intervals and monitoring for adherence in heart failure outpatients: the REMADHE trial. Circ Heart Fail 2008;1:115-124.

27. DELANEY C, APOSTOLIDIS B. Pilot testing of a multicomponent home care intervention for older adults with heart failure: an academic clinical partnership. J Cardiovasc Nurs 2010;25:E27-40.

28. BLUE L, LANG E, MCMURRAY JJV, DAVIE AP, MCDONAGH TA, MURDOCH DR, et al. Randomised controlled trial of specialist nurse intervention in heart failure. BMJ. 2001;323:715-718.

29. FERRANTE D, VARINI S, MACCHIA A, SOIFER $S$, BADRA R, NUL D, et al. Long-term results after a telephone intervention in chronic heart failure: DIAL (Randomized Trial of Phone Intervention in Chronic Heart Failure) follow-up. J Am Coll Cardiol 2010;56:372-378.

30. CLARKE M, SHAH A, SHARMA U. Systematic review of studies on telemonitoring of patients with congestive heart failure: a meta-analysis. J Telemed Telecare 2011;17:7-14.

31. CAPOMOLLA S, FEBO O, CERESA M, CAPOROTONDI A, GUAZZOTTI G, LA ROVERE MT, et al. Cost/utility ratio in chronic heart failure: comparison between heart failure management program delivered by day-hospital and usual care. J Am Coll Cardiol. 2002;40:1259-1266.

32. GARY RA, DUNBAR SB, HIGGINS MK, MUSSELMAN DL, SMITH AL. Combined exercise and cognitive behavioral therapy improves outcomes in patients with heart failure. J Psychosom Res 2010;69:119-131.

33. ANDERSEN K, JONSDOTTIR S, SIGURETHSSON AF, SIGURETHSSON SB. The effect of physical training in chronic heart failure. Laeknabladid 2006;92:759-764.

34. MICHE E, ROELLEKE E, ZOLLER B, WIRTZ U, SCHNEIDER M, HUERST $M$, et al. A longitudinal study of quality of life in patients with chronic heart failure following an exercise training program. Eur J Cardiovasc Nurs 2009;8:281-287.

35. VAN TOL BA, HUIJSMANS RJ, KROON DW, SCHOTHORST M, KWAKKEL G. Effects of exercise training on cardiac performance, exercise capacity and quality of life in patients with heart failure: a meta-analysis. Eur J Heart Fail 2006;8:841-850.

36. PINA IL. Exercise and heart failure: A statement from the American Heart Association Committee on exercise, rehabilitation, and prevention. Circulation 2003;107:1210-1225.

37. WANG TJ, EVANS JC, BENJAMIN EJ, LEVY D, LEROY EC, VASAN RS. Natural History of Asymptomatic Left Ventricular Systolic Dysfunction in the Community. Circulation. 2003;108:977-982.

38. RODRIGUEZ-ARTALEJO F, GUALLAR-CASTILLON P, PASCUAL CR, OTERO CM, MONTES AO, GARCIA AN, et al. Health-related quality of life as a predictor of hospital readmission and death among patients with heart failure. Arch Intern Med 2005;165:1274-1279.

39. STEWART S. Financial aspects of heart failure programs of care. European Journal of Heart Failure. 2005;7:423 -428.

40. INGLIS SC, PEARSON S, TREEN S, GALLASCH T, HOROWITZ JD, STEWART S. Extending the Horizon in Chronic Heart Failure: Effects of Multidisciplinary, HomeBased Intervention Relative to Usual Care. Circulation. 2006;114:2466-2473. 